\title{
Measurement of bandgap energies in low-k organosilicates
}

\author{
M. T. Nichols, ${ }^{1}$ W. Li, ${ }^{1}$ D. Pei, ${ }^{1}$ G. A. Antonelli, ${ }^{2}$ Q. Lin, ${ }^{3}$ S. Banna,,${ }^{4}$ Y. Nishi, ${ }^{5}$ \\ and J. L. Shohet ${ }^{1}$ \\ ${ }^{1}$ Plasma Processing and Technology Laboratory and Department of Electrical and Computer Engineering, \\ University of Wisconsin-Madison, Madison, Wisconsin 53706, USA \\ ${ }^{2}$ Lam Research, Tualatin, Oregon 97062, USA \\ ${ }^{3}$ T.J. Watson Research Center, IBM Corporation, Yorktown Heights, New York 10598, USA \\ ${ }^{4}$ Applied Materials, Sunnyvale, California 94085, USA \\ ${ }^{5}$ Department of Electrical Engineering, Stanford University, Stanford, California 94305, USA
}

(Received 7 January 2014; accepted 23 February 2014; published online 6 March 2014)

\begin{abstract}
In this work, experimental measurements of the electronic band gap of low-k organosilicate dielectrics will be presented and discussed. The measurement of bandgap energies of organosilicates will be made by examining the onset of inelastic energy loss in core-level atomic spectra using X-ray photoelectron spectroscopy. This energy serves as a reference point from which many other facets of the material can be understood, such as the location and presence of defect states in the bulk or at the interface. A comparison with other measurement techniques reported in the literature is presented. (C) 2014 AIP Publishing LLC.
\end{abstract}

[http://dx.doi.org/10.1063/1.4867644]

\section{INTRODUCTION}

In this work, experimental measurements of the electronic band gap of low-k organosilicate dielectrics will be presented and discussed. The measurement of bandgap energies of organosilicates will be made by examining the onset of inelastic energy loss in core-level atomic spectra using X-ray photoelectron spectroscopy (XPS). ${ }^{1}$ This energy serves as a reference point from which many other facets of the material can be understood, such as the location and presence of defect states in the bulk or at the interface. ${ }^{2}$

XPS is used to analyze the binding energies of the atomic core-level electrons in a material. These core-level spectra are representative of the chemical composition of the material near the surface (i.e., within the electron-escape depth) and, among other things, can give detailed information about changes in the atomic structure near the surface due to processing-induced damage. In addition to measuring the photoelectron intensities of core-level peaks, XPS can also be used to analyze the inelastic collisions that occur during photoexcitation and photoemission of electrons from the sample. These inelastic processes include band-to-band electronic transitions and excitation of "plasma waves" by Coulomb interaction with other electrons below the Fermi level, or equivalently for insulators and semiconductors (assuming there are no defect states below the Fermi level), with electrons in the valence band. As will be shown shortly, analysis of these inelastic processes can be used to determine the bandgap energy near the surface of the dielectric.

According to the three-step photoemission model, ${ }^{3}$ a photo-emitted electron in XPS undergoes three distinct transitions after photoexcitation but before reaching the electron analyzer. These processes are as follows: (1) the electron is excited by an incoming (X-ray) photon, (2) the electron is transported to the surface of the material, and (3) the electron escapes from the surface of the material into the vacuum. During step (2), a fast-moving charged particle can lose energy in the bulk material to collective high-frequency plasma oscillations of electrons in the valence band. The quasiparticles resulting from the quantization of these plasma oscillations are called plasmons, and the minimum energy of a plasmon excited in the bulk is given by $\hbar \omega_{p}=\sqrt{4 \pi \hbar^{2} N_{e} e^{2} / m_{e}}$, where $\omega_{p}$ is the plasma frequency in the bulk, $m_{e}$ is the free electron mass, and $N_{\mathrm{e}}$ is the density of electrons in the valence band. ${ }^{4}$ Because most solids have a valence band density of $10^{22}-10^{24}$ electrons $/ \mathrm{cm}^{3}$, typical plasmon energies are on the order of 4 to $30 \mathrm{eV}$. For a detailed derivation of the electron interactions leading to the energy loss spectrum discussed here, see Ref. 5.

Additionally, inelastic scattering may occur at the surface, i.e., during step (3), where the escaping electron can excite collective oscillations known as surface plasmons. An electron that excites a surface plasmon "loses" energy equal to the surface plasmon energy $\hbar \omega_{s}=\hbar \omega_{p} / \sqrt{2}$, where $\omega_{s}$ is the plasma frequency at the surface. Single-particle excitations due to band-to-band transitions (i.e., exciting another electron from the valence band into the conduction band) can occur during either step (2) or (3) and result in a third inelastic loss mechanism with a corresponding singleparticle energy loss of $\Delta \mathrm{E}_{s p}=E_{f}-E_{i}$, where $\mathrm{E}_{\mathrm{f}}$ is the energy of the final state of the excited electron and $E_{i}$ is its initial state. ${ }^{5}$ Because single-particle excitations involve the promotion of an electron from a filled state to an empty state of higher energy, in thermal equilibrium, these excitations must excite an electron from below the Fermi level to above it. For insulators or wide-bandgap intrinsic semiconductors, this is equivalent to the condition that an electron must be excited from the valence band into the conduction band. Hence, the fundamental lower limit of inelastic loss is equal to the bandgap energy. As will be discussed shortly, the presence of filled states (e.g., due to dopants or defects) above the valence band edge can also contribute to this signal, but since the density of such states is typically smaller than the 
density of electrons in the valence band, these states typically act to "smear" the signal corresponding to the onset of inelastic losses.

These inelastic collisions act to reduce the final kinetic energy of the photoexcited electron measured by the XPS detector. We can write the final kinetic energy of an electron having lost energy by all three inelastic mechanisms as $E_{k}=h \nu-E_{B}-\hbar \omega_{p}-\hbar \omega_{s}-\Delta \mathrm{E}_{s p}$, where $h \nu$ is the X-ray photon energy, $E_{B}$ is the original binding energy of the electron, and the other terms are as defined above. Certainly not every excited electron undergoes inelastic collisions before being detected, so the primary energy of interest (i.e., the binding energy $E_{B}$ ) can still be determined by conventional XPS. ${ }^{1}$

However, if a sufficient number of electrons lose energy due to these inelastic processes, photoelectron energy peaks with decreased kinetic energy relative to the core level can be experimentally observed. ${ }^{6}$ These "loss-spectra" peaks will appear as broadened copies of the core-level peaks shifted to lower kinetic energy. Because most commercial XPS software converts kinetic energy to binding energy automatically, in practice, peaks due to inelastic collisions will appear to have increased binding energy relative to their original level. The exact position of the peaks will depend on the magnitude and mechanism of energy loss, and the onset of the inelastic energy loss spectra corresponds directly to the bandgap energy. ${ }^{6}$

Sections II-VI describe the results of using this method for determination of the bandgap energies of organosilicate dielectrics. Confirmation of the efficacy of this method will be initially achieved by measurement of the bandgap of thermally grown $\mathrm{SiO}_{2}$. The method will then be used to measure the bandgap energies of low-k organosilicate films including UV-cured and as-deposited $\mathrm{SiCOH}$ as well as photopatternable low-k materials.

\section{SILICON DIOXIDE}

In order to measure the spectra due to inelastic energy loss, a suitable primary photoelectron peak must be chosen. Although in principle, any core-level spectra will exhibit the same inelastic losses, the measured photoelectron intensity must be large enough so that a sufficient signal-to-noise ratio can be achieved with suitably high resolution. Consequently, a preliminary survey scan of the sample must be performed to determine the primary photoelectron peaks present across a wide range of energies. Using XPS, survey scans for all samples were made using a pass-energy of $57 \mathrm{eV}$ (resulting in an absolute resolution of $2 \mathrm{eV}$ ) and a step size of $1 \mathrm{eV}$. A non-monochromatic $\mathrm{MgK} \alpha_{1,2}$ doublet $\mathrm{X}$-ray source with a centroid photon energy $h \nu=1253.603 \mathrm{eV}$ was used for all measurements. ${ }^{7}$ The results of a survey scan for a $100-\mathrm{nm}$ thick $\mathrm{SiO}_{2}$ sample are shown in Figure 1 .

The essential feature of this survey scan is the observation of a high-intensity core-level photoelectric peak, which in this case is due to $\mathrm{O} 1 \mathrm{~s}$ core-level electrons. As mentioned in the preceding paragraph, inelastic losses can be observed as a low-intensity core-level peak shifted to higher binding energies. Also observed in the figure are primary elastic

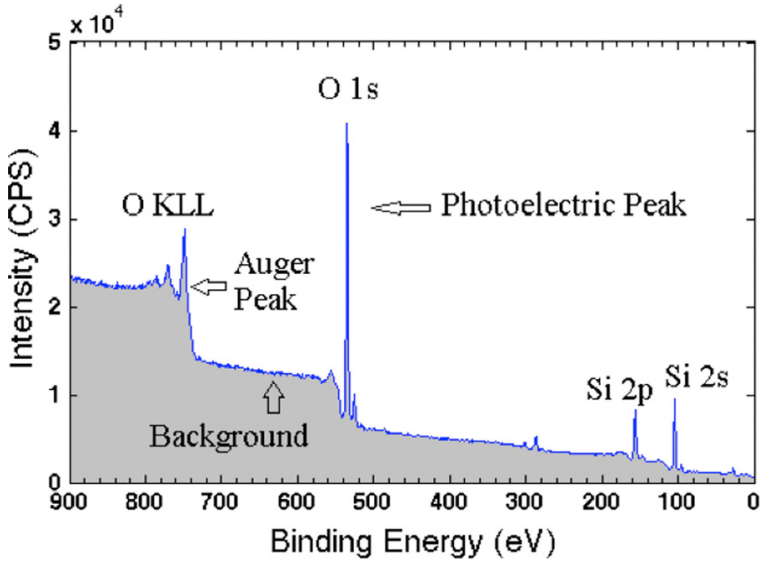

FIG. 1. XPS survey for thermally grown $\mathrm{SiO}_{2}$ showing primary photoelectron peaks, Auger peaks, and background intensity attributed to bremsstrahlung.

peaks due to Si 2p and Si 2s binding energies at lower intensities with corresponding (small) inelastic loss peaks. Three distinct peaks due to production of Auger electrons can be observed at high binding energy and are labeled as "KLL" peaks because of the core level hole due to photoemission (K-shell, 1s orbital) and the initial states of the two Auger electrons (L-shell, $2 \mathrm{p}$ orbital). The background signal due to bremsstrahlung is also shown as the gray shaded area. In Secs. III and IV, a Shirley background fitting ${ }^{8}$ is applied to all XPS spectra, and the background level is used as the zero-intensity reference.

A high-resolution scan of the peak with the largest observed intensity is shown in Figure 2. All high-resolution measurements were performed by selecting a pass energy of $21 \mathrm{eV}$ with a corresponding absolute resolution of $0.5 \mathrm{eV}$. Decreasing the pass energy will increase the resolution of the energy analyzer at the expense of significantly decreased signal intensity. Because the resolution of the XPS system used in this work is inherently limited by the finite linewidth of the $\mathrm{MgK} \alpha \mathrm{X}$-ray source $(\sim 0.7 \mathrm{eV})$, increasing the electron-detector resolution further would only result in a degraded signal-to-noise ratio. For $\mathrm{SiO}_{2}$, the largest signal corresponds to the primary photoelectron peak due to the oxygen 1s core level electrons located at $533.6 \mathrm{eV}$ binding energy. Because they tend to have higher cross sections for

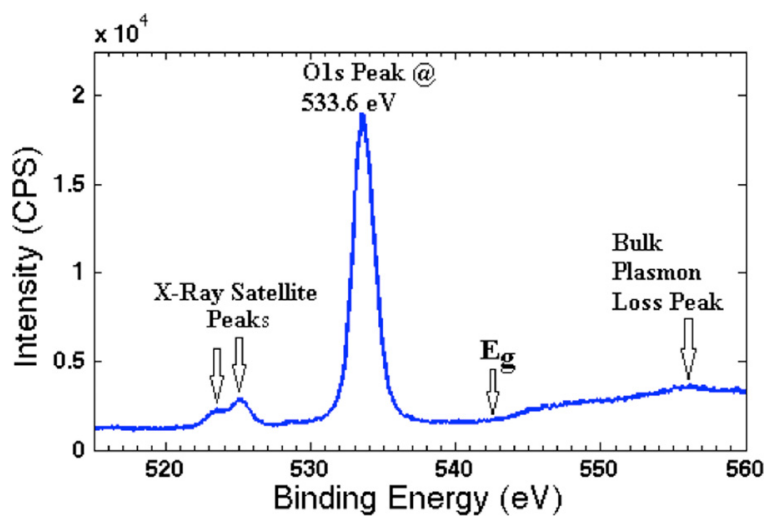

FIG. 2. XPS spectra for the $\mathrm{O} 1 \mathrm{~s}$ peak in $\mathrm{SiO}_{2}$. Note the horizontal axis is in increasing binding energy units. 
absorption of X-ray photons, photoelectron peaks at higher binding energies typically have a correspondingly larger photoelectron intensity (for a given atomic concentration). Hence, the $\mathrm{O}$ 1s peak is dominant for both $\mathrm{SiO}_{2}$ and for the organosilicate films to be discussed in this work.

As can be seen in Figure 2, the bulk plasmon loss peak is observed at approximately $556.1 \mathrm{eV}$, corresponding to a bulk plasmon energy $\hbar \omega_{p}=556.1 \mathrm{eV}-\mathrm{E}_{\mathrm{O} 1 \mathrm{~s}}=22.5 \mathrm{eV}$. A smaller inelastic peak is observed at $\sim 550 \mathrm{eV}$ binding energy with a loss energy equal to $15.4 \mathrm{eV}$. This peak is attributed to excitation of a surface plasmon resonance, which has an expected plasmon energy of $\hbar \omega_{s}=\hbar \omega_{p} / \sqrt{2}=15.91 \mathrm{eV}$. Additionally, two small overlapping peaks measured at $\sim 525 \mathrm{eV}$ (i.e., lower binding energy) can be attributed to X-ray satellite peaks at 8.4 and $10 \mathrm{eV}$ due to $\mathrm{K} \alpha_{3}$ and $\mathrm{K} \alpha_{4}$ transitions from the non-monochromatic $\mathrm{Mg} \mathrm{X}$-ray source. ${ }^{9}$ Because of the atomic structure of magnesium, no satellite peaks from this source can occur in the region of interest for studying the inelastic loss spectra. The approximate onset of inelastic losses corresponding to the band-gap energy is labeled in the figure as $E_{g}$.

To find the band-gap energy, a linear fit is made to the measured loss spectra curve near the approximate location of onset of inelastic losses. ${ }^{10}$ Next, by subtracting the Shirley background fitting, the background "zero" level is determined. The energy corresponding to the onset of inelastic losses is found by extrapolating the linear-fit line and calculating its intersection with the "zero" level. The bandgap energy is equal to the difference between the core-level peak energy and the onset of inelastic losses. This procedure is shown in Figure 3 for a $100-\mathrm{nm}$ thick film of thermally grown $\mathrm{SiO}_{2}$. The location of the O 1s elastic peak was determined to be $533.6 \mathrm{eV}$ as shown in Figure 2. A straight line is fit to the loss spectra; this fit is shown as the solid line in Figure 3. The background level for this measurement was calculated using AugerScan commercial software and is plotted as the horizontal dashed curve in the figure. The intersection of these two lines was calculated to occur at $542.4 \mathrm{eV}$. Thus, the bandgap energy is calculated as $E_{g}=E_{\text {loss }}-E_{O 1 s}=8.8 \mathrm{eV}$. This result is in excellent agreement with the established value of $\sim 8.9 \mathrm{eV} .^{11}$

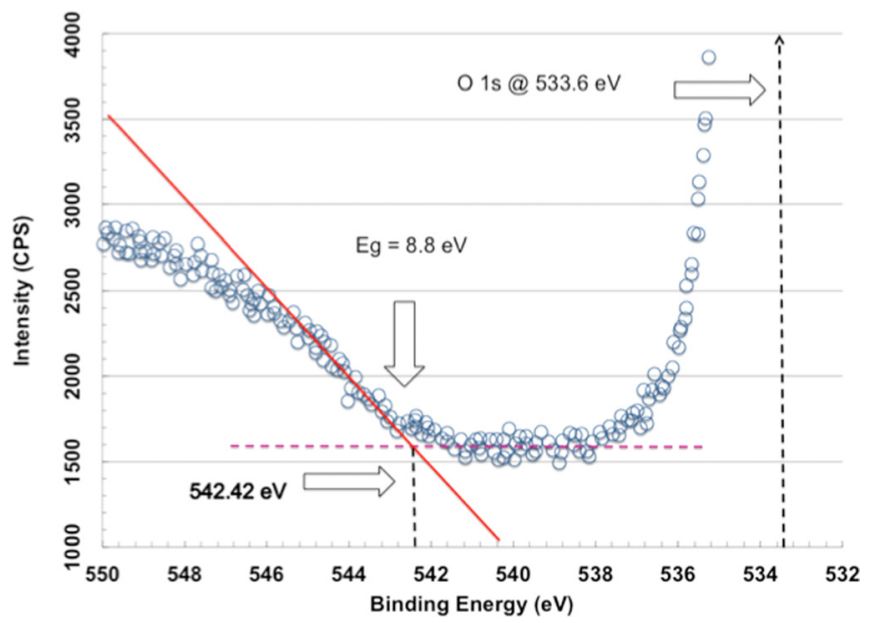

FIG. 3. Measurement of the bandgap of $\mathrm{SiO}_{2}$ using onset of electron energy loss spectra.

\section{ORGANOSILICATE FILMS}

By measuring the onset of inelastic loss relative to the $\mathrm{O}$ 1s core level peak, the bandgap energies for organosilicate films can also be determined. To further verify the efficacy of fitting a linear extrapolation about the inelastic loss peak, bandgap energies were measured for $644 \mathrm{~nm} \mathrm{k}=2.75$ asdeposited SiCOH films using the linear-extrapolation method as well as with an alternate method using commercial XPS curve-fitting software (CasaXPS). Results from the latter method are shown in Figure 4. This software automatically determines the peak onset by fitting a modified GaussianLorentzian curve to the inelastic loss spectra. Using this software, the onset energy was determined to be $543.8 \mathrm{eV}$. Subtracting this from the $\mathrm{O} 1 \mathrm{~s}$ peak at $537.1 \mathrm{eV}$, a bandgap energy of $E_{g}=6.7 \mathrm{eV}$ was computed.

Extrapolating a linear fit to the loss curve as was done with $\mathrm{SiO}_{2}$ yielded a bandgap energy of $\mathrm{E}_{\mathrm{g}}=7.0 \mathrm{eV}$. Although these two numbers are within the margin of error of the measurement of $\pm 0.7 \mathrm{eV}$, it is hypothesized that the linear-fit method is more accurate. This is due to the fact that defect states have been shown to exist near the valence-band edge of carbon-containing $\mathrm{SiO}_{2}$ using reflected energy-loss spectroscopy. ${ }^{12}$ Specifically, defects in these carbon-containing oxide films have been observed at $7.4 \mathrm{eV}$ and $7.6 \mathrm{eV}$ binding energies, produced by oxygen vacancies and carbon dangling bonds, respectively. ${ }^{12}$ Because the density of these defects is low relative to the density of electrons in the valence band, in practice, these defects can result in the onset of the loss peak appearing to be "smeared" out towards lower binding energy rather than appearing as a sharp transition. Hence, the bandgap measured by the commercial software is more likely to underestimate $\mathrm{E}_{\mathrm{g}}$ than the linear-fit method.

For the as-deposited films, the observed inelastic loss spectra were too smooth to discern the loss maxima produced at the onset of plasmon resonance. Additionally, despite the fact that data collection was averaged over a period of hours, the onset of the loss spectra was still unexpectedly indistinct. We hypothesize that this is due to the fact that the density of electrons in the valence band of the

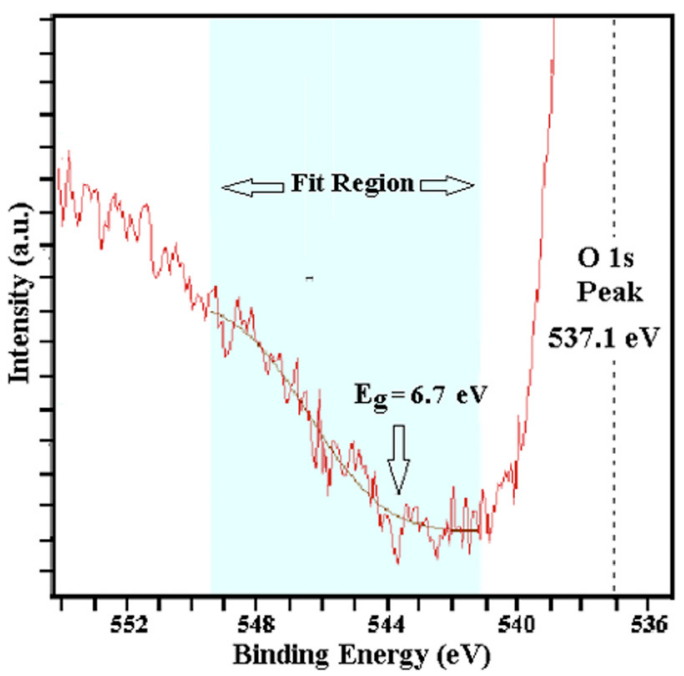

FIG. 4. Bandgap measurement for as-deposited SiCOH using commercial software. 
as-deposited film varies as a function of position across the sample surface. Since the $\mathrm{x}$-ray source used in this work illuminates an area of approximately $1 \mathrm{~mm}^{2}$, the measured photoelectron energies are sampled over the entire area. Hence, lateral variations in the electron density in the valence band near the surface will result in a measured spectrum that is the convolution of the different band energies.

Next, this method is applied to 500-nm thick UV-cured $\mathrm{SiCOH}$ films with $\mathrm{k}=2.65$. Figure 5 shows the onset of inelastic losses near the $\mathrm{O} 1 \mathrm{~s}$ core level. It can be observed that the data show significant qualitative improvement compared to the as-deposited samples, and considerably less measurement time was needed to obtain a satisfactorily high signal-to-noise ratio. For the UV-cured films measured, although no distinct peak could be attributed to surface plasmons, peaks attributed to the onset of bulk plasmon resonance could be clearly identified at binding energy of $556.0 \mathrm{eV}$. This corresponds to a bulk plasmon energy of $\hbar \omega_{p}=22.1 \mathrm{eV}$. Interestingly, this value is quite close to the value of $\hbar \omega_{p}$ $=22.5 \mathrm{eV}$ observed for $\mathrm{SiO}_{2}$, implying that the density of electrons in the valence band (and hence, the valence band structure) is quite similar for the two materials. $\mathrm{For}_{\mathrm{SiO}_{2}}$, the valence band structure is comprised primarily of $\mathrm{O} 2 \mathrm{p}$ nonbonding and $(\mathrm{O} 2 \mathrm{p}-\mathrm{Si} 3 \mathrm{p})$ or $(\mathrm{O} 2 \mathrm{p}-\mathrm{Si} 3 \mathrm{~s})$ bonding states. ${ }^{13}$ Although the low-k organosilicates like $\mathrm{SiCOH}$ contain a significant fraction of carbon ( $\sim 30 \%$ atomic concentration for this film), the carbon atoms have been verified using FTIR to exist predominantly as non-crosslinked $\mathrm{CH}_{\mathrm{x}}$ groups (i.e., bonded primarily to one silicon or one oxygen atom only), ${ }^{14}$ and consequently, since the remaining $\mathrm{Si}$ and $\mathrm{O}$ bonds are highly crosslinked (through $\mathrm{O} 2 \mathrm{p}-\mathrm{Si} 3 \mathrm{p}$ and $\mathrm{O} 2 \mathrm{p}-\mathrm{Si} 3$ s bonding states), it is reasonable to believe that the valence band structure is of similar composition to that of $\mathrm{SiO}_{2}$.

By fitting a straight line to the inelastic loss spectra, shown as the solid line in Figure 5, and extrapolating this to intersect with the background level (dashed horizontal line), the onset of inelastic losses is found to occur at $541.6 \mathrm{eV}$. Using the measured $\mathrm{O} 1 \mathrm{~s}$ energy of $533.9 \mathrm{eV}$, the bandgap energy was calculated to be $E_{g}=7.7 \mathrm{eV}$.

Despite the fact that this procedure of determining bandgap energies has been demonstrated to be in very good

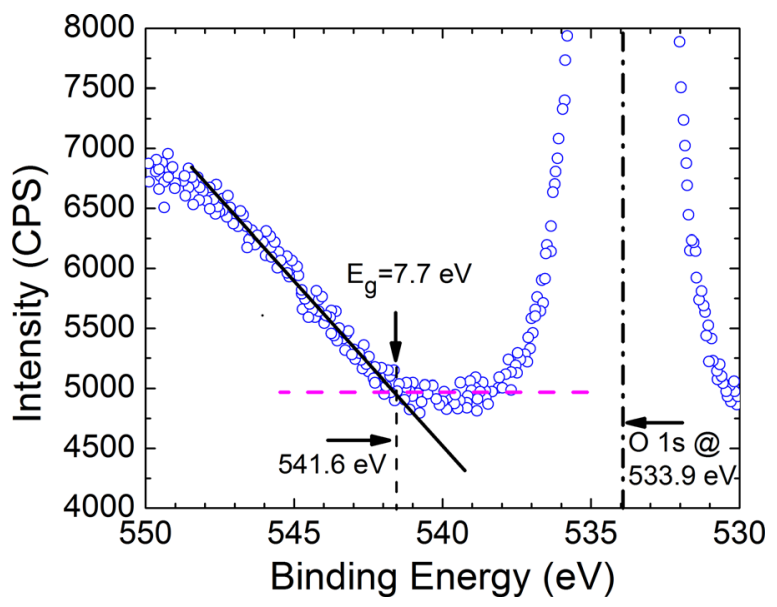

FIG. 5. Bandgap measurements of UV-cured SiCOH showing linear fit to inelastic absorption (solid line) and background level (horizontal dotted line). agreement with reported values for $\mathrm{SiO}_{2}$, error bounds on the calculated value must be also considered. For the line-fitting method, the largest source of error is due to uncertainty in the process of linear extrapolation (i.e., extending the line past the measured spectra data).

In order to quantify this uncertainty, Figure 6 shows the 95\% confidence intervals in the linear regression (least squares fit) to the inelastic loss peak. For this plot, the x-axis corresponds to the electron energy loss, which is calculated by subtracting the measured energies from the $\mathrm{O} 1 \mathrm{~s}$ elastic peak energy. In this way, the energy lost due to inelastic processes can be read directly from the axis.

By taking the upper and lower bounds of the confidence intervals and finding their intersection with the background level, it is possible to find upper and lower error bounds on the band gap calculations. According to this analysis, we can determine with $95 \%$ confidence that the bandgap energy lies between $7.2 \mathrm{eV}$ and $8.2 \mathrm{eV}$; in other words, the bandgap $\mathrm{E}_{\mathrm{g}}=7.7 \pm 0.5 \mathrm{eV}$ with $95 \%$ certainty.

\section{PHOTOPATTERNABLE LOW-K DIELECTRIC FILMS}

Because photopatternable low-k materials share essentially the same chemical structure as the $\mathrm{SiCOH}$ films analyzed in this work, it may be reasonable to expect that their bandgap energies are similar. To verify this hypothesis, bandgap measurements were made on $324 \mathrm{~nm}, \mathrm{k}=2.75 \mathrm{UV}$ cured PPLK films. The results of this analysis are shown in Figure 7. As with UV-cured $\mathrm{SiCOH}$, the $\mathrm{O} 1$ s core level is used as a reference point, and is observed to be at $534.20 \mathrm{eV}$. As before, a straight line (shown as the solid line in the figure) is fit to the observed inelastic peak, and the intersection of this line with the background level (dashed horizontal line) is found. The intersection point was calculated to be $542.45 \mathrm{eV}$, corresponding to a bandgap energy of $\mathrm{E}_{\mathrm{g}}=8.25 \mathrm{eV}$. To determine the uncertainty in this value, 95\% confidence intervals are constructed from the linear regression, and their intersections with the background level are calculated. This gives bandgap energies between 7.75 and $8.75 \mathrm{eV}$, which is equivalent to $\mathrm{E}_{\mathrm{g}}=8.25 \pm 0.5 \mathrm{eV}$ with 95\% confidence.

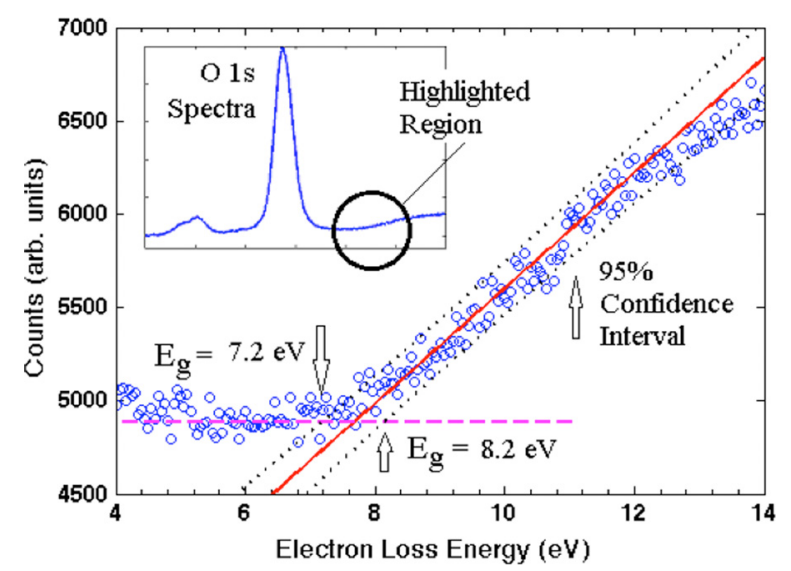

FIG. 6. Electron energy loss spectra showing linear fit (solid line) with 95\% confidence interval (dotted lines) for UV-cured $\mathrm{SiCOH}$. Inset: region of $\mathrm{O} 1 \mathrm{~s}$ spectra corresponding to loss spectra. Note the horizontal axis is in units of increasing electron loss energy. 


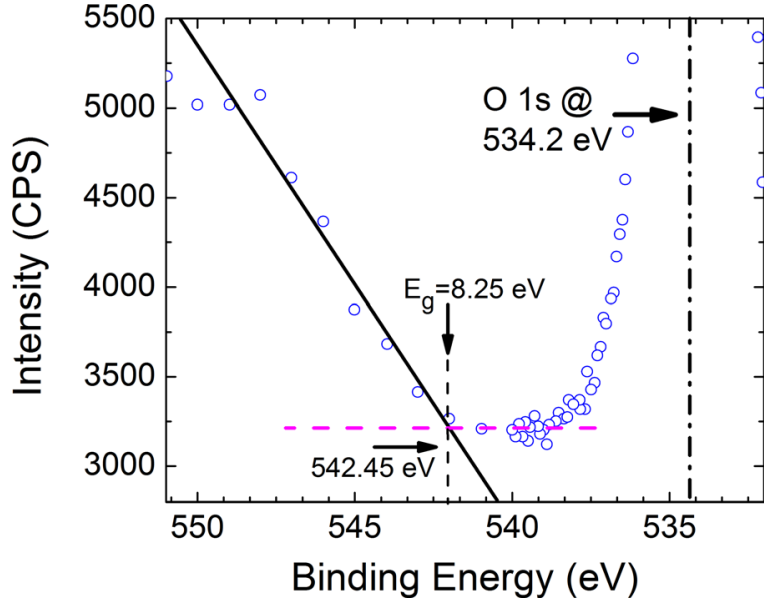

FIG. 7. Bandgap measurements for $\mathrm{k}=2.75$ UV-cured PPLK materials showing $95 \%$ confidence interval.

\section{LEAKAGE CURRENTS}

It has been suggested that the level of leakage currents could also be representative of the bandgap. Figure 8 shows the leakage currents for as-deposited and UV-cured $\mathrm{SiCOH}$. The bandgap for as-deposited $\mathrm{SiCOH}$ was measured to be $7.2 \mathrm{eV}$ and that for $\mathrm{UV}$-cured $\mathrm{SiCOH}$ was measured to be $7.7 \mathrm{eV}$. The leakage current in as-deposited $\mathrm{SiCOH}$ is always higher than in UV-cured $\mathrm{SiCOH}$ and it also has a lower breakdown voltage. The leakage currents in these low-k films ( $\sim 500 \mathrm{~nm}$ thick) are primarily caused by the presence of defects or impurities in the dielectric, some of which are present in the bandgap. These defect concentration levels more typically appear as a result of processing procedures and are likely responsible for the leakage currents, rather than having them caused by tunneling through the bandgap itself.

\section{COMPARISON WITH OTHER REPORTS OF THE BANDGAP ENERGY}

As has been mentioned previously, the knowledge of the bandgap energy provides a necessary energy reference for understanding the depths of trap states with the band gap as

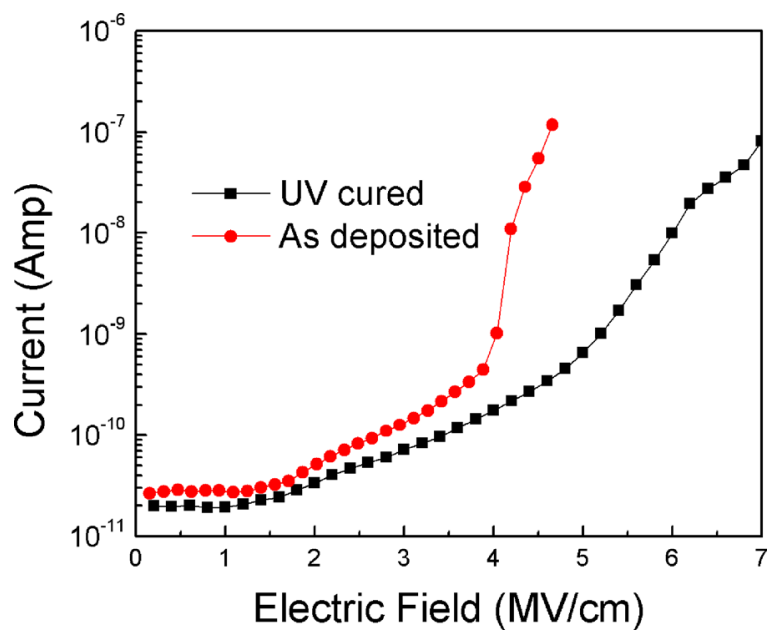

FIG. 8. Comparison of leakage currents versus electric field for as-deposited and UV-cured $\mathrm{SiCOH}$. well as interfacial energy-barrier heights. Because the conduction mechanisms in low-k dielectrics are fundamentally dependent on accurate knowledge of these energies, discrepancies in the apparent bandgap energies between different measurement techniques are quite significant. Hence, it is worth discussing the organosilicate bandgap measurements made in this work within the context of other available literature on the subject.

Most past references concerning the band gap of organosilicate films come from the work of Grill and his group. ${ }^{15}$ This group was responsible for the initial invention and subsequent development of carbon-doped oxide materials deposited by PECVD, which includes low-k SiCOH. According to their work, the band gap in organosilicate films is strongly dependent on the plasma parameters used for film deposition and on the fraction of carbon added to reduce the dielectric constant. For "early" dense low-k films, these researchers cited bandgap energies between 4.74 and $5.92 \mathrm{eV}$ depending on the dielectric constant. ${ }^{16}$ Interestingly, the band gap was not found to scale linearly with the dielectric constant. Later investigations, which focused on films with higher porosity content and lower k-values, cite even smaller gap energies. ${ }^{17}$ These ranged from $2.91 \mathrm{eV}$ to $3.62 \mathrm{eV}$ and were found to be proportional to the concentration of $\mathrm{CH}_{\mathrm{x}}$ groups added during the deposition process (i.e., inversely proportional to $\mathrm{k}$-value). ${ }^{17}$ Adding to this confusion, other researchers claim to show that the band gap energy was equal to $9 \mathrm{eV}$, which is more than $66 \%$ larger than the next largest cited value found in the literature. ${ }^{18}$ The results of the bandgap measurements from these studies and from the present work are summarized in Table I.

To compare these past results to those from this work, their methods of determining the band gap energy must first be discussed. According to the Grill group, ${ }^{19}$ film thicknesses, optical properties, and band gaps in the above cited papers were measured using a spectrometric reflectance tool, specifically the N\&K model. ${ }^{19}$ According to the manufacturer, the range of wavelengths used by this tool is between 190 and $1000 \mathrm{~nm}$, and it calculates optical constants from reflectance data using the Forouhi-Bloomer relations. Indeed, the Forouhi-Bloomer (F-B) equations are quite suitable for optical property calculations from reflectance measurements made on amorphous materials. ${ }^{20}$ The F-B equations, which determine the index of refraction and extinction coefficient as a function of photon energy, are derived from the quantum theory of absorption of light, have a direct dependence on the material bandgap (and hence, the bandgap can be calculated from experimental data), and can be shown to satisfy the Kramers-Kronig relations. Hence, when used appropriately, these equations can provide a physically rigorous determination of the optical properties of amorphous dielectric films. ${ }^{20}$

However, as noted by others, the F-B equations were originally derived for use in the interband energy region of a material. ${ }^{21}$ In other words, they were meant to be applied to measured data that is taken using photon energies larger than the bandgap energy. Since the wavelength range of the $\mathrm{N} \& \mathrm{~K}$ analyzer used in the above-cited work is limited to a maximum photon energy of $6.53 \mathrm{eV}$ (190 nm wavelength), 
TABLE I. Bandgap measurements in low-k organosilicates.

\begin{tabular}{|c|c|c|c|c|}
\hline Dielectric & $\mathrm{K}$ & $\mathrm{Eg}(\mathrm{eV})$ & Method & Reference \\
\hline Dense $\mathrm{SiCOH}$ & 3.99 & 4.74 & Optical reflectance & Grill (1999), Ref. 16 \\
\hline Dense $\mathrm{SiCOH}$ & 3.25 & 5.92 & Optical reflectance & Grill (1999), Ref. 16 \\
\hline Porous $\mathrm{SiCOH}$ & 2.6 & 2.91 & Optical reflectance & Grill (2003), Ref. 17 \\
\hline Porous $\mathrm{SiCOH}$ & 2.1 & 3.62 & Optical reflectance & Grill (2003), Ref. 17 \\
\hline Porous $\mathrm{SiCOH}$ & 2.55 & 9.0 & VUV spectroscopy & Lauer (2010), Ref. 18 \\
\hline Porous $\mathrm{SiCOH}$ & 2.65 & $7.7 \pm 0.5$ & XPS & This work \\
\hline Porous $\mathrm{SiCOH}$ & $2.3,2.8$ & $8.0 \pm 0.4$ & REELS & King (2013), Ref. 24 \\
\hline As-deposited SiCOH & 2.75 & 7.2 & XPS & This work \\
\hline PPLK & 2.75 & $8.25 \pm 0.5$ & XPS & This work \\
\hline Thermal $\mathrm{SiO}_{2}$ & 3.9 & 8.8 & XPS & This work \\
\hline
\end{tabular}

the vast majority of the measured optical data correspond to photon energies lower than their bandgap measurements of $\sim 6 \mathrm{eV}$.

Hence, it is plausible that the light source used in previous work by the Grill group was not capable of emitting short-enough wavelengths for accurate determination of the optical bandgap energy of the films they studied. In fact, other work has confirmed this precise shortcoming of the F-B model. In one instance, optical-property measurements of a thin film of amorphous silicon nitride were made over the wavelength range of $240-1000 \mathrm{~nm} .^{22}$ The optical data were then analyzed using three separate models: the F-B model mentioned above, the Lorenz model, and the TaucLorenz model. ${ }^{22}$ In each of the three cases, film thicknesses and optical constants showed good agreement with each other, indicating that certain properties can be accurately determined using the limited wavelength range. However, despite this apparent agreement, the F-B model was found to dramatically underestimate the bandgap of the sample material, giving a value of $2.85 \mathrm{eV}$ (with high uncorrelated error) compared to the value of $4.3 \mathrm{eV}$ (with small uncorrelated error) from the Tauc-Lorentz model (note that the Lorenz model does not explicitly include bandgap energies). The latter value is in good agreement with the expected value of amorphous silicon nitride films, which have been confirmed using a number of methods to lie between $4.5 \mathrm{eV}$ and $5.3 \mathrm{eV}$ depending on film composition. $^{23}$

Given the above considerations, it is possible that the claims of $<6 \mathrm{eV}$ band-gap energies stem from a possible misapplication of an otherwise physically sound model, despite the fact that it can still provide accurate thickness results even with a relatively poor estimate of the bandgap. Additional verification of the results presented here, based on the onset of electron energy loss, comes from very recent work $^{24,25}$ using the method of reflected electron-energy-loss spectroscopy (REELS) in a Auger electron-spectroscopy system. Their work confirms a bandgap of approximately $8.0 \pm 0.4 \mathrm{eV}$ for $\mathrm{UV}$-cured $\mathrm{SiCOH}$ (irrespective of dielectric constant). This value falls well within the margin of error for the bandgap of $7.7 \pm 0.5 \mathrm{eV}$ presented in this work. Hence, there is good evidence to assert the validity of the methods used here leading to the conclusion the correct bandgap energy is $\sim 7.7 \mathrm{eV}$.

\section{SUMMARY}

In this work, analysis of the inelastic loss spectrum in core-level XPS spectra was used to determine the bandgap energies of low-k organosilicate dielectrics. This method was verified by first measuring the bandgap energy for thermally grown $\mathrm{SiO}_{2}$ films, which were determined to be $\mathrm{E}_{\mathrm{g}}=8.8 \mathrm{eV}$ in good agreement with past literature. The linear-extrapolation method was compared with fitting the loss spectra to a Gaussian-Lorentzian using commercial XPS software. The latter was hypothesized to underestimate bandgap energies due to the presence of defects near the valence-band edge. Gap energies of $7.2 \mathrm{eV}$ were measured for as-deposited films, $8.25 \mathrm{eV}$ for UV-cured photopatternable low-k films, and for UV-cured $\mathrm{SiCOH}$ films a bandgap energy of $E_{g}=7.7 \mathrm{eV}$ was found. Relative confidence in the bandgap measurements was established by constructing $95 \%$ confidence intervals based on the inelastic loss spectra linear regression and extrapolation. Using these confidence intervals, the bandgap energy of UV-cured $\mathrm{SiCOH}$ was found to be $7.7 \mathrm{eV} \pm 0.5 \mathrm{eV}$ with $95 \%$ certainty.

Because this energy is of fundamental importance for determination of leakage-current processes in these dielectrics and in order to determine defect-state energies within the bandgap and obtain an estimate of band alignments and barrier energies with other materials, in-depth analysis of the existing (and potentially conflicting) measurements was undertaken. After the analysis, it was hypothesized that some of the previously cited lower values can be due to a slight misinterpretation of the Forouhi-Bloomer equations for calculating bandgap energy from measured reflectance data.

\section{ACKNOWLEDGMENTS}

The authors wish to express their thanks to S. W. King of Intel Corporation for helpful suggestions and comments. This work was supported by the National Science Foundation under Grant No. CBET-1066231, by the Semiconductor Research Corporation under Contract No. 2012-KJ-2359 and by the Applied Materials University Research Partnership Program.

${ }^{1}$ D. Penn, "Theory of the electron energy-loss spectrum in core-level X-ray photoemission from solids," Phys. Rev. Lett. 38, 1429 (1977). 
${ }^{2}$ S. Sze and K. Ng, Physics of Semiconductor Devices (Wiley, 2006), Vol. 1. ${ }^{3}$ S. Hügner, Photoelectron Spectroscopy: Principles and Applications (Springer, 2003).

${ }^{4}$ D. Pines, "Collective energy losses in solids," Rev. Mod. Phys. 28, 184 (1956)

${ }^{5}$ P. Nozieres and D. Pines, "Electron interaction in solids. Characteristic energy loss spectrum," Phys. Rev. 113, 1254 (1959)

${ }^{6} \mathrm{~F}$. Bell and L. Ley, "Photoemission study of $\mathrm{SiO}_{x(0<x<2)}$ alloys," Phys. Rev. B 37, 8383 (1988).

${ }^{7}$ J. Schweppe, R. Deslattes, T. Mooney, and C. Powell, "Accurate measurement of $\mathrm{Mg}$ and $\mathrm{Al} \mathrm{K} \alpha_{1,2} \mathrm{X}$-ray energy profiles," J. Electron Spectrosc. Relat. Phenom. 67, 463 (1994).

${ }^{8}$ S. Tougaard and C. Jansson, "Comparison of validity and consistency of methods for quantitative XPS peak analysis," Surf. Interface Anal. 20, 1013 (1993).

${ }^{9} \mathrm{C}$. Klauber, "Refinement of magnesium and aluminum $\mathrm{K} \alpha \mathrm{X}$-ray source functions," Surf. Interface Anal. 20, 703 (1993).

${ }^{10}$ S. Miyazaki, "Characterization of high-k gate dielectric/silicon interfaces," Appl. Surf. Sci. 190, 66 (2002).

${ }^{11}$ T. DiStefano and D. Eastman, "The band edge of amorphous $\mathrm{SiO}_{2}$ by photoinjection and photoconductivity measurements," Solid State Commun. 9, 2259 (1971).

${ }^{12}$ P. Poveda and A. Glachant, "Energy gap-determination of a carbon contaminated thermal silicon oxide thin film using reflection electron energy loss spectroscopy," J. Non-Cryst. Solids 216, 83 (1997).

${ }^{13}$ B. Fischer, R. Pollak, T. DiStefano, and W. Grobman, "Electronic structure of $\mathrm{SiO}_{2}, \mathrm{Si}_{x} \mathrm{Ge}_{1-x} \mathrm{O}_{2}$, and $\mathrm{GeO}_{2}$ from photoemission spectroscopy," Phys. Rev. B 15, 3193 (1977).

${ }^{14}$ E. Andideh, M. Lerner, G. Palmrose, S. El-Mansy, T. Scherban, G. Xu, and J. Blaine, "Compositional effects on electrical and mechanical properties in carbon-doped oxide dielectric films: Application of Fouriertransform infrared spectroscopy,” J. Vac. Sci. Technol. B 22, 196 (2004).
${ }^{15}$ A. Grill and D. A. Neumayer, "Structure of low dielectric constant to extreme low dielectric constant $\mathrm{SiCOH}$ films: Fourier transform infrared spectroscopy characterization,” J. Appl. Phys. 94, 6697 (2003).

${ }^{16}$ A. Grill, L. Perraud, V. Patel, C. Jahnes, and S. Cohen, Low-Dielectric Constant SiCOH Films as Potential Candidates for Interconnect Dielectrics (Mater. Res. Soc. Symp. Proc., Cambridge University Press, 1999), Vol. 565, p. 107.

${ }^{17}$ A. Grill, "Plasma enhanced chemical vapor deposited $\mathrm{SiCOH}$ dielectrics: From low-k to extreme low-k interconnect materials," J. Appl. Phys. 93, 1785 (2003).

${ }^{18}$ J. Lauer, H. Sinha, M. Nichols, G. Antonelli, Y. Nishi, and J. Shohet, "Charge trapping within UV and vacuum UV irradiated low- $\kappa$ porous organosilicate dielectrics," J. Electrochem. Soc. 157, G177 (2010).

${ }^{19}$ A. Grill and V. Patel, "Ultralow- $\kappa$ dielectrics prepared by plasmaenhanced chemical vapor deposition," Appl. Phys. Lett. 79, 803 (2001).

${ }^{20} \mathrm{~A}$. Forouhi and I. Bloomer, "Optical dispersion relations for amorphous semiconductors and amorphous dielectrics," Phys. Rev. B 34, 7018 (1986).

${ }^{21}$ D. Poelman and P. Smet, "Methods for the determination of the optical constants of thin films from single transmission measurements: a critical review," J. Phys. D 36, 1850 (2003).

${ }^{22} \mathrm{H}$. Tompkins and E. Irene, Handbook of Ellipsometry (William Andrew, 2005), p. 271.

${ }^{23}$ A. Iqbal, W. Jackson, C. Tsai, J. Allen, and C. Bates, "Electronic structure of silicon nitride and amorphous silicon/silicon nitride band offsets by electron spectroscopy," J. Appl. Phys. 61, 2947 (1987).

${ }^{24}$ S. W. King, B. French, and E. Mays, "Detection of defect states in low-k dielectrics using reflection electron energy loss spectroscopy," J. Appl. Phys. 113, 044109 (2013)

${ }^{25}$ B. French and S. W. King, "Detection of surface electronic defect states in low- and high-k dielectrics using reflection electron-energy-loss spectroscopy,” J. Mater. Res. 28, 2771 (2013). 